\title{
Lot-Order Assignment Applying Priority Rules for the Single-Machine Total Tardiness Scheduling with Nonnegative Time-Dependent Processing Times
}

\author{
Jae-Gon Kim, ${ }^{1}$ Seung-Kil Lim, ${ }^{2}$ and June-Young Bang ${ }^{2}$ \\ ${ }^{1}$ Department of Industrial and Management Engineering, Incheon National University, 12-1 Songdo-dong, Yeonsu-gu, \\ Incheon 406-840, Republic of Korea \\ ${ }^{2}$ Department of Industrial and Management Engineering, Sungkyul University, 400-10 Anyang-dong, Manan-gu, Anyang, \\ Gyeonggi-do 430-742, Republic of Korea
}

Correspondence should be addressed to June-Young Bang; jybang@sungkyul.ac.kr

Received 21 January 2015; Accepted 13 March 2015

Academic Editor: Yunqiang Yin

Copyright (C) 2015 Jae-Gon Kim et al. This is an open access article distributed under the Creative Commons Attribution License, which permits unrestricted use, distribution, and reproduction in any medium, provided the original work is properly cited.

\begin{abstract}
Lot-order assignment is to assign items in lots being processed to orders to fulfill the orders. It is usually performed periodically for meeting the due dates of orders especially in a manufacturing industry with a long production cycle time such as the semiconductor manufacturing industry. In this paper, we consider the lot-order assignment problem (LOAP) with the objective of minimizing the total tardiness of the orders with distinct due dates. We show that we can solve the LOAP optimally by finding an optimal sequence for the single-machine total tardiness scheduling problem with nonnegative time-dependent processing times (SMTTSPNNTDPT). Also, we address how the priority rules for the SMTTSP can be modified to those for the SMTTSP-NNTDPT to solve the LOAP. In computational experiments, we discuss the performances of the suggested priority rules and show the result of the proposed approach outperforms that of the commercial optimization software package.
\end{abstract}

\section{Introduction}

Lot-order assignment is to assign items in lots being processed to orders to fulfill the orders in make-to-order production systems. Typical lot-order assignment examples include assigning wafers in lots to orders in a semiconductor wafer fabrication facility and assigning panels in lots to orders in a color-filter fabrication facility of the thin film transistor liquid crystal display industry. In general, an order is satisfied with several lots because the order size is typically larger than the lot size; however, a lot may be used to satisfy several orders also. Additional lots should be released into the production facility if all the orders cannot be satisfied with lots currently in the production facility. Lot-order assignment is as important as production planning and scheduling for meeting the due dates of orders (especially in a manufacturing industry with a long production cycle time such as the semiconductor manufacturing industry). Lot-order assignment is usually performed periodically with a one-day or one-shift cycle before production begins.

Compared to the production planning and scheduling problem, there have only been a few studies on lot-order assignment problems (LOAPs), most of which have involved the semiconductor manufacturing industry. Knutson et al. [1], Fowler et al. [2], Carlyle et al. [3], Boushell et al. [4], and $\mathrm{Ng}$ et al. [5] studied lot-order assignment problems in semiconductor assembly and test facilities, whereas $\mathrm{Wu}[6]$, Bang et al. [7], and Kim and Lim [8] studied these problems in wafer fabrication facilities. Kim and Lim [8] provided insight into the characteristics of the LOAP. They suggested the compact pegging method and showed that the LOAP becomes equivalent to an order sequence problem with this compact pegging method. On the other hand, Lim et al. [9] developed a Lagrangian heuristic for simultaneous orderlot pegging and release planning in semiconductor wafer fabrication facilities. 
The existing algorithms for the LOAP are not practical since they are not easy to implement and require long computation time. In this study, we show that the LOAP can solve optimally by finding an optimal sequence for the singlemachine total tardiness scheduling problem with nonnegative time-dependent processing times (SMTTSP-NNTDPT). Also, we address how the priority rules for the SMTTSP can be modified to those for the SMTTSP-NNTDPT to solve the LOAP.

\section{Mathematical Formulation}

The LOAP considered in this paper is to determine the assignment of items in lots to orders in a production facility, as well as a plan to release additional lots into the production facility (when needed) with the objective of minimizing the total tardiness of the orders over a finite time horizon. To simplify the problem, it is assumed that items in any lot can be assigned to any order. This assumption is realistic in many cases where lots and orders can be classified into different groups according to their product types. Thus, lots and orders of the same product type can be dealt with separately for lotorder assignments. In addition, we assume that new lots are released into the production facility on a daily basis (when needed) and lot-order assignment is performed with a oneday cycle before production begins. Further, it is assumed that the current day is represented as "day 0" and the current day's production begins at time zero without loss of generality. We use the following notation for modeling the problem mathematically:

$N$ : number of orders,

$L$ : number of lots in process,

$T$ : length of the time horizon (day),

$i$ : index for order,

$l$ : index for lot,

$t$ : index for time period,

$q_{i}$ : quantity of order $i$,

$w_{l}$ : number of items in lot $l$,

$Q_{t}$ : limit on the total number of items that can be released into the production facility at day $t$,

$r_{l}$ : remaining processing time of lot $l$ (hour),

$d_{i}$ : due date of order $i$ (hour),

CT: production cycle time (hour),

$C_{i}$ : completion time of order $i$ (hour),

$\tau_{i}$ : tardiness of order $i$ (hour),

$x_{i l}$ : number of items in lot $l$ that are assigned to order $i$,

$y_{i t}$ : number of items to be newly released at day $t$ for order $i$,

$z_{i l}$ : equal to 1 if lot $l$ is used to satisfy order $i, 0$ otherwise,

$u_{i t}$ : equal to 1 if new lots are released at day $t$ for order $i, 0$ otherwise.
Now, we present a mixed integer linear programming formulation of the LOAP:

$$
\begin{aligned}
& \text { [LOAP] Minimize } \sum_{i=1}^{N} \tau_{i} \\
& \text { subject to } \sum_{l=1}^{L} x_{i l}+\sum_{t=1}^{T} y_{i t}=q_{i} \quad \forall i \\
& \sum_{i=1}^{N} x_{i l} \leq w_{l} \quad \forall l \\
& \sum_{i=1}^{N} y_{i t} \leq Q_{t} \quad \forall t \\
& x_{i l} \leq w_{l} z_{i l} \quad \forall i, l \\
& y_{i t} \leq Q_{t} u_{i t} \quad \forall i, t \\
& C_{i} \geq r_{l} z_{i l} \quad \forall i, l \\
& C_{i} \geq(24 t+\mathrm{CT}) u_{i t} \quad \forall i, t \\
& \tau_{i} \geq C_{i}-d_{i} \quad \forall i \\
& \tau_{i} \geq 0 \quad \forall i \\
& x_{i l} \geq 0 \quad \forall i, l \\
& y_{i t} \geq 0 \quad \forall i, t \\
& z_{i l} \in\{0,1\} \quad \forall i, l \\
& u_{i t} \in\{0,1\} \quad \forall i, t .
\end{aligned}
$$

The objective function (1) to be minimized denotes the total tardiness of all orders. Constraint (2) ensures that order quantity should be satisfied with existing lots and/or newly released ones. Constraint (3) limits the maximum number of items in a lot that can be assigned to orders. Constraint (4) limits the maximum number of items that can be released at each period. Constraint (5) specifies the number of items in each lot which are assigned to orders. Constraint (6) specifies the number of items which are newly released in each time period for orders. Note that the number of lots newly released in each period can be automatically determined given the number of items newly released in each period and the lot sizes, although this is not included in the model. Constraints (7) and (8) specify the completion time of each order. Constraint (9) specifies the tardiness of each order. Constraints (10) to (14) represent decision variables.

Figure 1 shows a lot-order assignment for a small example with four orders and four lots. In the figure, $x_{11}=20, x_{12}=$ $10, x_{22}=10, x_{23}=5, x_{33}=10, x_{34}=20, y_{40}=25$, and the total tardiness is nine. Note that 25 new items should be released at day 0 (current day) to satisfy order 4 in this case. 


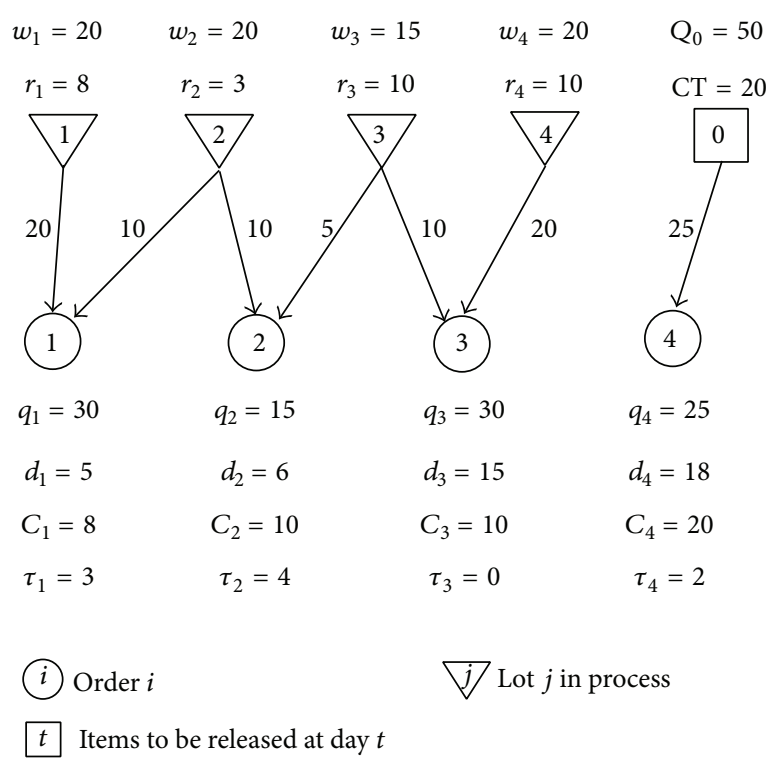

FiguRE 1: Illustration of a lot-order assignment.

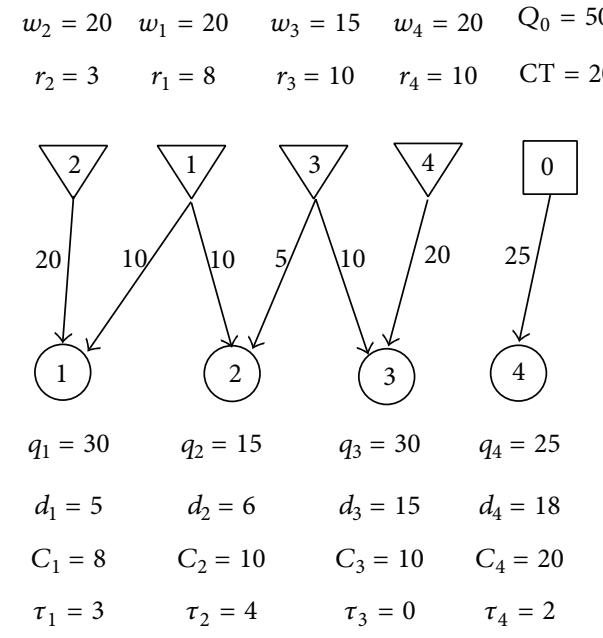

(a) Order sequence: 1, 2,3,4

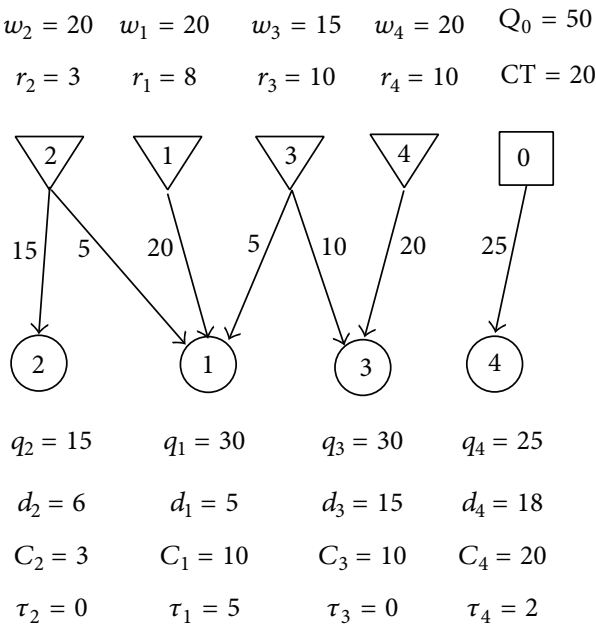

(b) Order sequence: 2, 1, 3, 4

FIGURE 2: Lot-order assignments using the compact pegging method for two order sequences.

\section{Relationship between the LOAP and the SMTTSP-NNTDPT}

Kim and Lim [8] proved that the LOAP is strongly NPhard and proposed a lot-order assignment method called the compact pegging method which generates the best lot-order assignment with the minimum total tardiness for a given order sequence for assigning items in lots to orders. In the compact pegging method, items (in lots) with less remaining time are assigned to orders in earlier positions in the order sequence. Figure 2 shows two lot-order assignments for the example, which were obtained by applying the compact pegging method to two order sequences. Lots are lined up in increasing order of the remaining time, and they are assigned to orders one by one according to the order sequence. In the figure, the order sequence 2, 1, 3, 4 (Figure 2(b)) yields a better result than the order sequence 1, 2, 3, 4 (Figure 2(a)) in terms of total tardiness (7 versus 9).

Kim and Lim [8] proved that there exists an (optimal) order sequence from which an optimal solution (lot-order assignment) for the LOAP can be obtained using the compact pegging method. Thus, the LOAP becomes equivalent to the problem of finding an optimal order sequence for assigning items in lots to orders. In this section, we show that we can obtain an optimal order sequence for the LOAP by solving the SMTTSP with nonnegative time-dependent processing times. As a result, we can solve the LOAP optimally by finding an optimal sequence for the SMTTSP with nonnegative timedependent processing times. 
Scheduling with time-dependent processing times means that the job processing times can be changed by the time when the job is started. Many researches, including Cheng et al. ([10], survey paper), Cheng et al. [11], Yin et al. [12], and Yin and $\mathrm{Xu}$ [13], consider the cases in which processing times are changed by deterioration or learning effect. In real production systems, processing time including setup times can be changed with sequence (position) of jobs. There are a number of studies on scheduling problems with timedependent processing time based on sequence-dependent setup times, as surveyed in Allahverdi et al. [14]. Recently, Hung et al. [15] consider scheduling problems with semiconductor multi-head testers in which the processing time is related to the product mix. However, there are few researches which deal with the scheduling problems with nonnegative time-dependent processing times. In this section, we suggest some properties of the LOAP and prove that the optimal solution can be obtained by finding the optimal sequence for SMTTSP-NNTDPT.

Let $C_{i}^{\pi}$ be the completion time of order $i$ in order sequence $\pi$ when the compact pegging method is applied to order sequence $\pi$, and let $C(\cdot)$ be the total completion time for orders in order set $\cdot$, that is, the required time to complete all orders in order set $\cdot$.

Property 1. For any order set $\Omega, C(\Omega)$ is constant regardless of the order sequence.

Proof. $C(\Omega)$ is equal to the completion time of the lot with the longest remaining time among the lots assigned to the orders in $\Omega$. In the compact pegging method, items in lots are assigned to orders in an increasing order of the remaining processing times of the lots until the total demand of the orders is satisfied. Because the total demand of the orders is constant regardless of the order sequence, the last lot assigned to an order is not changed. Thus, the total completion time of the orders is constant regardless of their sequence (e.g., the total completion time is 20 for any order sequence in Figure 2).

Property 2. For any two sets of orders $\Omega_{1}$ and $\Omega_{2}$, the total completion times for $\Omega_{1}$ and $\Omega_{2}$ have the following relationships:

$$
\begin{aligned}
& \text { (1) if } \sum_{i \in \Omega_{1}} q_{i}=\sum_{i \in \Omega_{2}} q_{i} \text {, then } C\left(\Omega_{1}\right)=C\left(\Omega_{2}\right) \text {, } \\
& \text { (2) if } \sum_{i \in \Omega_{1}} q_{i}<\sum_{i \in \Omega_{2}} q_{i} \text {, then } C\left(\Omega_{1}\right) \leq C\left(\Omega_{2}\right) .
\end{aligned}
$$

Proof. The relationship (1) holds because the last lot assigned to the two sets of orders, $\Omega_{1}$ and $\Omega_{2}$, is the same when the two sets of orders have the same total number of items. The relationship (2) also holds because it may be necessary to assign additional lots with longer remaining times to the orders in $\Omega_{2}$ compared to ones in $\Omega_{1}$.

Property 3. If order $i$ precedes order $j$ in any order sequence $\pi$, then $C_{i}^{\pi} \leq C_{j}^{\pi}$.

Proof. In the lot-order assignment method, lots with less remaining times are assigned to orders at the earlier positions in the order sequence. Therefore, the lots assigned to order $j$ have the same or longer completion times than those assigned to order $i$.

We define the processing time of order $i$ in order sequence $\pi$ as the time duration to complete order $i$ after completing the preceding order in $\pi$ and denote it by $\rho_{i}^{\pi}$. Let $U_{i}^{\pi}$ be a set of orders which precede order $i$ in $\pi$, and let [i] be an index of the $i$ th order in an order sequence. Then, $\rho_{[i]}^{\pi}$ is given as below:

$$
\rho_{[i]}^{\pi}=C_{[i]}^{\pi}-C_{[i-1]}^{\pi} \quad \forall i .
$$

Property 4. Consider $C_{i}^{\pi}=C\left(U_{i}^{\pi} \cup\{i\}\right)$.

Proof. By definition of $C(\cdot), C\left(U_{i}^{\pi} \cup\{i\}\right)=\max _{j \in U_{i}^{\pi} \cup\{i\}} C_{j}^{\pi}$. By Property $3, \max _{j \in U_{i}^{\pi}} C_{j}^{\pi} \leq C_{i}^{\pi}$. It follows that $C\left(U_{i}^{\pi} \cup\{i\}\right)=$ $C_{i}^{\pi}$.

By Property 4, (15) can be rewritten as

$$
\rho_{i}^{\pi}=C\left(U_{i}^{\pi} \cup\{i\}\right)-C\left(U_{i}^{\pi}\right) \quad \forall i .
$$

Property 5. If $q_{i} \geq q_{j}$ and $U_{i}^{\pi}=U_{j}^{\pi^{\prime}}, \rho_{i}^{\pi} \geq \rho_{j}^{\pi^{\prime}}$.

Proof. It is obvious by Properties 2 and 4 .

Property 6. The completion time of the last order in any order sequence is equal to the total completion time of the orders; that is, $C_{[N]}^{\pi}=C(\Omega)$ for any $\pi$.

Proof. It is obvious by Properties 1 and 4.

Property 7. The sum of the processing times of all orders for any order sequence $\pi$ is equal to the total completion time of the orders; that is, $\sum_{i \in \Omega} \rho_{i}^{\pi}=C(\Omega)$.

Proof. Consider $\sum_{i \in \Omega} \rho_{i}^{\pi}=C_{[1]}^{\pi}+\left(C_{[2]}^{\pi}-C_{[1]}^{\pi}\right)+\left(C_{[3]}^{\pi}-C_{[2]}^{\pi}\right)+$ $\cdots+\left(C_{[N]}^{\pi}-C_{[N-1]}^{\pi}\right)=C_{[N]}^{\pi}=C(\Omega)$.

Property 8. The processing times of orders are timedependent.

Proof. The completion times of orders are determined by both the order quantities and the lots assigned to the orders. The lots assigned to each order are determined by the sequence of the orders. Therefore, the processing times of orders are time-dependent.

Property 9. The processing times of orders are nonnegative.

Proof. By Property 3 and (15), the completion time of order $[i]$ is greater than that of order $[i-1]$; that is, $C_{[i]}^{\pi} \geq C_{[i-1]}^{\pi}$, so that, $\rho_{[i]}^{\pi}=C_{[i]}^{\pi}-C_{[i-1]}^{\pi} \geq 0$.

Properties 8 and 9 can be easily verified in the example shown in Figure 2. In Figure 2, $\rho_{1}^{\pi}=8, \rho_{2}^{\pi}=2, \rho_{3}^{\pi}=0$, $\rho_{4}^{\pi}=10$, and $\rho_{1}^{\pi^{\prime}}=3, \rho_{2}^{\pi^{\prime}}=7, \rho_{3}^{\pi^{\prime}}=0, \rho_{4}^{\pi^{\prime}}=10$, where $\pi=(1,2,3,4)$ and $\pi^{\prime}=(2,1,3,4)$. Note that the processing time of order 3 becomes zero as shown in Figure 2, although the processing time is strictly greater than zero 


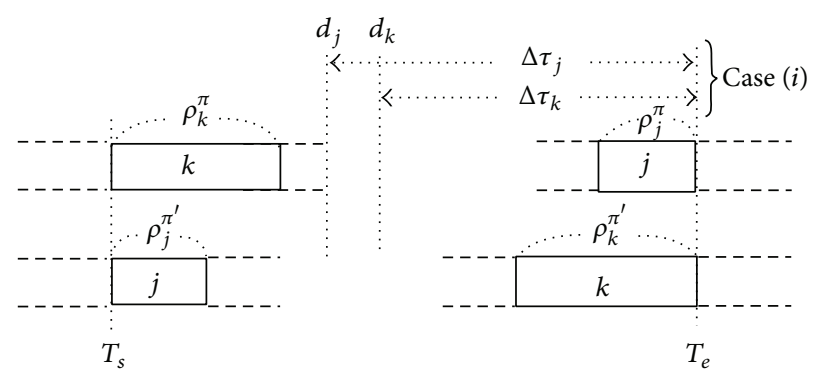

Figure 3: The effect of interchanging two orders in an order sequence in Property 10.

in a typical SMTTSP. This is a distinct characteristic not considered in previous research on the scheduling problem with time-dependent processing times.

Theorem 1. An optimal solution for the LOAP can be obtained by finding an optimal sequence for the SMTTSP with nonnegative time-dependent processing times (SMTTSP-NNTDPT).

Proof. By applying the compact pegging method to any order sequence $\pi$, we can obtain $\rho_{i}^{\pi}$ and $C_{i}^{\pi}$ for all $i$. According to Properties 8 and $9, \rho_{i}^{\pi}$ is time-dependent and nonnegative. If we consider $\rho_{i}^{\pi}$ and $C_{i}^{\pi}$ as the processing time and the completion time of job $i$ in the SMTTSP-NNTDPT, respectively, finding an optimal order sequence for the LOAP becomes equivalent to the problem of finding an optimal job sequence for the SMTTSP-NNTDPT.

\section{Priority Rules for SMTTSP-NNTDPT}

Because the SMTTSP-NNTDPT is NP-hard, it is considerably difficult to obtain an optimal solution when the problem size is large, similar to the SMTTSP. In this paper, we suggest several priority rules for the SMTTSP-NNTDPT, which can be easily applied to practice. Priority rules are used to determine the order sequence to which the compact pegging method is applied to obtain lot-order assignments for the LOAP. Various priority rules exist for the SMTTSP, such as SPT, EDD, MDD, SLACK, COVERT, and ATC [16, 17]. In this section, we address how the priority rules for the SMTTSP can be modified to those for the SMTTSP-NNTDPT. Among the many priority rules for the SMTTSP, six priority rules (EDD, SPT, MDD, SLACK, COVERT, and ATC) are most widely used in practice and are known to perform better than others [18-21]. Therefore, these six priority rules are modified for the SMTTSP-NNTDPT in this paper.

4.1. EDD Rule. In the EDD rule, orders with earlier due dates have higher priorities and are considered before those with later due dates in an order sequence. Ties are broken by selecting the order with the minimum order quantity. The EDD rule can be simply represented by EDD: $\min \left(d_{i}\right)$. We derive the optimality property of the EDD rule for the SMTTSP- NNTDPT [22].
Property 10. If order quantities and due dates are agreeable for any pair of orders, total tardiness is minimized by EDD sequencing.

Proof. Consider order sequence $\pi$ in which order $k$ precedes order $j$ with $q_{j} \leq q_{k}$ and $d_{j} \leq d_{k}$. Let $T_{s}$ and $T_{e}$ be the times at which order $k$ starts and order $j$ ends, respectively (see Figure 3). Let $\pi^{\prime}$ be a sequence which is obtained by interchanging orders $j$ and $k$ in $\pi$. Because $q_{j} \leq q_{k}, \rho_{k}^{\pi} \geq \rho_{j}^{\pi^{\prime}}$ and $\rho_{j}^{\pi} \leq \rho_{k}^{\pi^{\prime}}$ according to Property 2 . Note that there exists no (in)equality relationship between $\rho_{j}^{\pi}$ and $\rho_{j}^{\pi^{\prime}}$ (or $\rho_{k}^{\pi}$ and $\rho_{k}^{\pi^{\prime}}$ ). We will show that interchanging the two orders must decrease, or possibly leave unchanged, the total tardiness. Clearly, all orders which precede order $k$ or follow order $j$ in the original sequence are unaffected when the two orders are interchanged. All orders between order $k$ and $j$ are advanced in time, which decreases or leaves unchanged their tardiness. The changes in tardiness of orders $j$ and $k$ are investigated by considering following three cases.

(i) If $d_{j} \leq d_{k}<T_{e}$, as illustrated in Figure 3, then the decrease of tardiness of order $j$ is $\Delta \tau_{j}=T_{e}-\max \left(T_{s}+\right.$ $\left.\rho_{j}^{\pi^{\prime}}, d_{j}\right)$, the increase of tardiness of order $k$ is $\Delta \tau_{k}=$ $T_{e}-\max \left(T_{s}+\rho_{k}^{\pi}, d_{k}\right)$, and the net decrease due to the tow changes is $\Delta \tau_{j}-\Delta \tau_{k}=\max \left(T_{s}+\rho_{k}^{\pi}, d_{k}\right)-\max \left(T_{s}+\right.$ $\left.\rho_{j}^{\pi^{\prime}}, d_{j}\right)$, which is nonnegative because $d_{k} \geq d_{j}$ and $\rho_{k}^{\pi} \geq \rho_{j}^{\pi^{\prime}}$.

(ii) If $d_{j} \leq T_{e} \leq d_{k}, \Delta \tau_{j}-\Delta \tau_{k}=\Delta \tau_{j} \geq 0$.

(iii) If $T_{e}<d_{j} \leq d_{k}, \Delta \tau_{j}=\Delta \tau_{k}=0$. Thus in all cases the total decrease of tardiness is positive, or at worst zero, so the change should be made.

4.2. MOQ Rule. The MOQ rule corresponds to the SPT rule for the SMTTSP; that is, the order with the shortest processing time has the highest priority. Although processing times of the SMTTSP-NNTDPT are nonnegative and timedependent, they can be replaced with the order quantities according to Property 5 when using the MOQ rule. Thus, the order with the minimum order quantity has the highest priority in the MOQ rule. Ties are broken by selecting an 


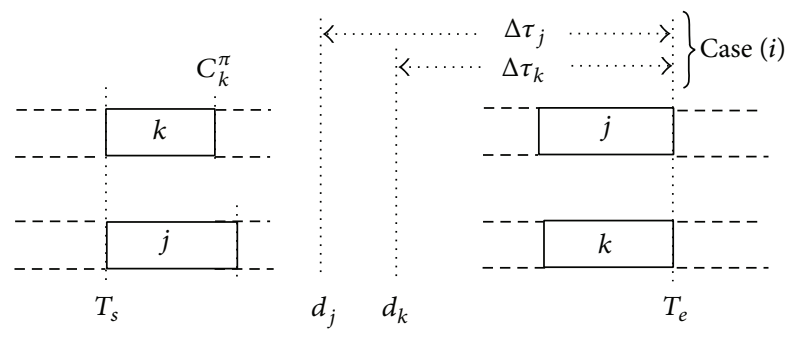

FIgURE 4: The effect of interchanging two orders in an order sequence in Property 11.

order with the earliest due date. The MOQ rule can be simply represented by MOQ: $\min \left(q_{i}\right)$.

4.3. MDD Rule. The MDD rule was originally suggested by Baker and Bertrand [23] for the SMTTSP. The MDD rule for the SMTTSP-NNTDPT can be modified based on Property 11 below.

Property 11. If order $k$ comes before order $j$ in order sequence $\pi$ and $\max \left(d_{k}, C_{k}^{\pi}\right)>\max \left(d_{j}, C_{j}^{\pi^{\prime}}\right), \pi^{\prime}$ gives less total tardiness than $\pi$, where $\pi^{\prime}$ is an order sequence in which positions of orders $j$ and $k$ are interchanged in $\pi$.

Proof. Assume that order $k$ starts at time $T_{s}$ and order $j$ is completed at time $T_{e}$ in $\pi$ as shown in Figure 4 and $\max \left(d_{k}, C_{k}^{\pi}\right)>\max \left(d_{j}, C_{j}^{\pi^{\prime}}\right)$. We will show that interchanging the two orders must decrease, or possibly leave unchanged, the total tardiness. Let $\tau_{j}^{\prime}$ and $\tau_{k}^{\prime}$ be tardiness of order $j$ and order $k$ in $\pi^{\prime}$, respectively. We can consider following four cases.

(i) Consider $\max \left(d_{j}, C_{j}^{\pi^{\prime}}\right)=d_{j}, \max \left(d_{k}, C_{k}^{\pi}\right)=d_{k}$, and $d_{j}<d_{k}$ as illustrated in Figure 4 . Then $\tau_{k}=\max \left(C_{k}^{\pi}-\right.$ $\left.d_{k}, 0\right)=0, \tau_{j}=\max \left(T_{e}-d_{j}, 0\right), \tau_{k}^{\prime}=\max \left(T_{e}-d_{k}, 0\right)$, and $\tau_{j}^{\prime}=\max \left(C_{j}^{\pi^{\prime}}-d_{j}, 0\right)=0$. The decrease of tardiness of order $j$ is $\Delta \tau_{j}=\max \left(T_{e}-d_{j}, 0\right)$ and the increase of that of order $k$ is $\Delta \tau_{k}=\max \left(T_{e}-d_{k}, 0\right)$. Because $d_{j}<d_{k}, \Delta \tau_{j} \geq \Delta \tau_{k}$.

(ii) Consider $\max \left(d_{j}, C_{j}^{\pi^{\prime}}\right)=C_{j}^{\pi^{\prime}}, \max \left(d_{k}, C_{k}^{\pi}\right)=d_{k}$, and $C_{j}^{\pi^{\prime}}<d_{k}$. Then $\tau_{k}=\max \left(C_{k}^{\pi}-d_{k}, 0\right)=0$, $\tau_{j}=\max \left(T_{e}-d_{j}, 0\right)=T_{e}-d_{j}, \tau_{k}^{\prime}=\max \left(T_{e}-d_{k}, 0\right)$, and $\tau_{j}^{\prime}=\max \left(C_{j}^{\pi^{\prime}}-d_{j}, 0\right)=C_{j}^{\pi^{\prime}}-d_{j}$. The decrease of tardiness of order $j$ is $\Delta \tau_{j}=T_{e}-C_{j}^{\pi^{\prime}}$ and the increase of that of order $k$ is $\Delta \tau_{k}=\max \left(T_{e}-d_{k}, 0\right)$. Because $C_{j}^{\pi^{\prime}}<d_{k}, \Delta \tau_{j}>\Delta \tau_{k}$.

(iii) Consider $\max \left(d_{j}, C_{j}^{\pi^{\prime}}\right)=d_{j}, \max \left(d_{k}, C_{k}^{\pi}\right)=C_{k}^{\pi}$, and $d_{j}<C_{k}^{\pi}$. Then $\tau_{k}=\max \left(C_{k}^{\pi}-d_{k}, 0\right)=C_{k}^{\pi}-d_{k}$, $\tau_{j}=\max \left(T_{e}-d_{j}, 0\right)=T_{e}-d_{j}, \tau_{k}^{\prime}=\max \left(T_{e}-d_{k}, 0\right)=$ $T_{e}-d_{k}$, and $\tau_{j}^{\prime}=\max \left(C_{j}^{\pi^{\prime}}-d_{j}, 0\right)=0$. The decrease of tardiness of order $j$ is $\Delta \tau_{j}=T_{e}-d_{j}$ and the increase of that of order $k$ is $\Delta \tau_{k}=T_{e}-C_{k}^{\pi}$. Because $d_{j}<C_{k}^{\pi}$, $\Delta \tau_{j}>\Delta \tau_{k}$.

(iv) Consider $\max \left(d_{j}, C_{j}^{\pi^{\prime}}\right)=C_{j}^{\pi^{\prime}}, \max \left(d_{k}, C_{k}^{\pi}\right)=C_{k}^{\pi}$, and $C_{k}^{\pi^{\prime}}<C_{k}^{\pi}$. Then $\tau_{k}=\max \left(C_{k}^{\pi}-d_{k}, 0\right)=C_{k}^{\pi}-d_{k}$, $\tau_{j}=\max \left(T_{e}-d_{j}, 0\right)=T_{e}-d_{j}, \tau_{k}^{\prime}=\max \left(T_{e}-d_{k}, 0\right)=$ $T_{e}-d_{k}$, and $\tau_{j}^{\prime}=\max \left(C_{j}^{\pi^{\prime}}-d_{j}, 0\right)=C_{j}^{\pi^{\prime}}-d_{j}$. The decrease of tardiness of order $j$ is $\Delta \tau_{j}=T_{e}-C_{j}^{\pi^{\prime}}$ and the increase of that of order $k$ is $\Delta \tau_{k}=T_{e}-C_{k}^{\pi}$. Because $C_{k}^{\pi^{\prime}}<C_{k}^{\pi}, \Delta \tau_{j}>\Delta \tau_{k}$.

We define the modified due date for an order as the larger value between the due date of the order and the earliest possible completion time of the order, which can be obtained by temporarily assigning lots with the least remaining time to the order until the order demand is satisfied. According to Property 11, orders with less modified due dates should be considered before ones with later modified due dates to minimize total tardiness. In the MDD rule, orders are selected in succession, and the $i$ th selected order is placed at the $i$ th position in the order sequence. When selecting an order, the order with the least modified due date among the unselected orders is selected. The modified due dates of orders should be updated each time a new order is selected because the earliest possible completion times of the unselected orders depend on those of selected ones. In this regard, the MDD rule is called the dynamic priority rule. MDD can be simply represented by MDD: $\min \left\{\max \left(d_{i}, t+\rho_{i}\right)\right\}$, where $t$ is the total completion time of the orders selected until now, and $\rho_{i}$ is the processing time of order $i$ in the SMTTSP-NNTDPT. Note that $t+\rho_{i}$ is the earliest possible completion time of order $i$ with the selected orders given. Because order sequence $\pi$ is not given but needs to be determined, we use notation $\rho_{i}$ instead of $\rho_{i}^{\pi}$ for convenience. Although $\rho_{i}$ is nonnegative and time-dependent, it can be calculated once the predecessors of order $i$ are given. Let $\Psi$ be the set of selected orders. Then $t=C(\Psi)$ and $\rho_{i}=C(\Psi \cup\{i\})-C(\Psi)$. Procedure 1 shows the steps for selecting orders and assigning lots to these orders in the MDD rule. 
TABLE 1: Summary of the priority rules.

\begin{tabular}{lcc}
\hline Rule & Definition & Rank and priority index \\
\hline EDD & Earliest due date & $\min \left(d_{i}\right)$ \\
\hline MOQ & Minimum order quantity & $\min \left(q_{i}\right)$ \\
\hline MDD & Modified due date & $\min \left\{\max \left(d_{i}, t+\rho_{i}\right)\right\}$ \\
\hline SLACK & Slack & $\min \left(d_{i}-t-\rho_{i}\right)$ \\
\hline COVERT & Cost over time & $\max \left\{\frac{1}{\rho_{i}} \max \left[0,1-\frac{\max \left(0, d_{i}-t-\rho_{i}\right)}{k \rho_{i}}\right\}\right\}$ \\
\hline ATC & Apparent tardiness cost & $\max \left\{\frac{1}{\rho_{i}} \exp \left(\frac{-\max \left(0, d_{i}-t-\rho_{i}\right)}{k \bar{\rho}}\right)\right\}$
\end{tabular}

Procedure 1 (MDD).

Step 0. Let $p=1, \Psi=\varnothing$, and $t=0$.

Step 1. For order $i \notin \Psi$, obtain $\rho_{i}$ by $\rho_{i}=C(\Psi \cup\{i\})-C(\Psi)$ and obtain the modified due date of the order, that is, $\max \left(d_{i}, t+\right.$ $\left.\rho_{i}\right)$.

Step 2. Select $i^{*}=\arg \min _{i \notin \Psi}\left\{\max \left(d_{i}, t+\rho_{i}\right)\right\}$ and place order $i^{*}$ at the $p$ th position in an order sequence. Let $\Psi \leftarrow \Psi \cup\left\{i^{*}\right\}$, $t \leftarrow t+C\left(\Psi \cup\left\{i^{*}\right\}\right)$, and $p \leftarrow p+1$. If $p \leq N$, go to step 1 .

Step 3. Assign lots to order $i^{*}$ using the compact pegging method.

4.4. SLACK Rule. In the SLACK rule, the order with the least slack time has the highest priority. The slack time of an order is defined as the maximum available time to delay the completion of the order without violating its due date. The order slack time is computed as the difference between the order due date and the earliest possible completion time for the order. As with the MDD rule, the SLACK rule is also a dynamic priority rule because the order slack time is not static but should be updated each time a new order is selected and lots are assigned to the order. The SLACK rule can be simply represented by SLACK: $\min \left(\mathrm{SLACK}_{i}\right)$, where $\mathrm{SLACK}_{i}=d_{i}-t-\rho_{i}$.

4.5. COVERT Rule. The original COVERT priority index represents the expected tardiness cost per unit of imminent processing time, or cost over time [17]. The COVERT rule is a ratio-based priority rule which combines the SLACK rule and the SPT rule. It puts the order with the largest COVERT ratio in the first position. The COVERT ratio of an order is computed by dividing a derived urgency ratio of the order by the processing time of the order. The COVERT rule can be simply represented by COVERT: $\max \left\{\left(1 / \rho_{i}\right) \max [0,1-\right.$ $\left.\left.\max \left(0, d_{i}-t-\rho_{i}\right) / k \rho_{i}\right]\right\}$, where $k$ is a parameter. The value of $k$ is usually determined through experimental analysis. The COVERT rule is also a dynamic priority rule. It is known that it performs well on the due date-based objectives, especially on the total tardiness for the SMTTSP [24].

4.6. ATC Rule. The ATC rule was developed based on the COVERT rule for the SMTTSP [17]. The basic concept of the ATC rule is the same with the COVERT rule with two main differences. First, the ATC rule uses an exponential function rather than linear one to emphasize the part of slack. Second, the ratio is calculated by dividing the order slack by the average processing time, instead of the order processing time. The ATC rule can be simply represented by ATC: $\max \left\{\left(1 / \rho_{i}\right) \exp \left(-\max \left(0, d_{i}-t-\rho_{i}\right) / k \bar{\rho}\right)\right\}$, where $k$ is a parameter and $\bar{\rho}=\sum_{i \notin \Psi} \rho_{i}$.

Table 1 summarizes the six priority rules for the SMTTSPNNTDPT. The first two rules, EDD and MOQ, are static rules, and the rest rules on the other hand are dynamic ones. In the static rules, the order priority index values do not change over time, whereas they might change over time in the dynamic rules.

\section{Computational Experiments}

To test the performances of the suggested priority rules, we randomly generated 540 problem instances of the LOAP while varying the number of orders, number of lots, and duedate tightness. We considered 27 combinations: three levels for the number of orders $(50,100$, and 200), three levels for the number of processing lots (sufficient, limited, and short), and three levels for the due-date tightness (loose, normal, and tight). We then generated 20 replication problem instances for each combination to obtain 540 problem instances. In each problem instance, the relevant data were generated as follows. Here, $D U(a, b)$ and $U(a, b)$ denote random numbers generated from the discrete and continuous distributions with a range $[a, b]$, respectively.

(1) The order size, $N$, was set to $D U(50,300)$ for each order.

(2) The production cycle time, CT, was set to DU $(150,200)$ hours.

(3) The remaining time of lot $l, r_{l}$, was set to CT $\cdot U(0,1)$ hours.

(4) The length of the time horizon was set to 4 weeks.

(5) The lot sizes were set to $D U(10,20)$ for each lot.

(6) $Q_{t}$ was set to 1000 units for all $t$.

(7) Due dates of orders were set to $\alpha$. CT for each order, where $\alpha$ is a due-date factor which is set to $1.7,1.3$, and 
TABLE 2: Results with CPLEX.

\begin{tabular}{|c|c|c|c|c|}
\hline $\begin{array}{l}\text { Number } \\
\text { of orders }\end{array}$ & $\begin{array}{l}\text { Due date } \\
\text { tightness }\end{array}$ & Lots & $\begin{array}{c}\text { Number of } \\
\text { optimal } \\
\text { solutions found }\end{array}$ & $\begin{array}{l}\text { Number of } \\
\text { feasible } \\
\text { solutions } \\
\text { found }\end{array}$ \\
\hline \multirow{9}{*}{50} & \multirow{3}{*}{ Loose } & Sufficient & 20 & 20 \\
\hline & & Limited & 4 & 20 \\
\hline & & Short & 0 & 20 \\
\hline & \multirow{3}{*}{ Normal } & Sufficient & 15 & 20 \\
\hline & & Limited & 0 & 20 \\
\hline & & Short & 0 & 20 \\
\hline & \multirow{3}{*}{ Tight } & Sufficient & 0 & 20 \\
\hline & & Limited & 0 & 20 \\
\hline & & Short & 0 & 20 \\
\hline \multirow{9}{*}{100} & \multirow{3}{*}{ Loose } & Sufficient & 16 & 20 \\
\hline & & Limited & 3 & 20 \\
\hline & & Short & 0 & 20 \\
\hline & \multirow{3}{*}{ Normal } & Sufficient & 12 & 20 \\
\hline & & Limited & 0 & 15 \\
\hline & & Short & 0 & 17 \\
\hline & \multirow{3}{*}{ Tight } & Sufficient & 0 & 0 \\
\hline & & Limited & 0 & 19 \\
\hline & & Short & 0 & 16 \\
\hline \multirow{9}{*}{200} & \multirow{3}{*}{ Loose } & Sufficient & 16 & 16 \\
\hline & & Limited & 0 & 0 \\
\hline & & Short & 0 & 1 \\
\hline & \multirow{3}{*}{ Normal } & Sufficient & 12 & 12 \\
\hline & & Limited & 0 & 0 \\
\hline & & Short & 0 & 0 \\
\hline & \multirow{3}{*}{ Tight } & Sufficient & 0 & 0 \\
\hline & & Limited & 0 & 0 \\
\hline & & Short & 0 & 0 \\
\hline
\end{tabular}

0.9 for generating loose, normal, and tight due dates, respectively.

(8) The number of processing lots was set to $[\beta$. (the sum of order sizes/average lot size)], where $\beta$ is a number of lots factor which is set to 1.0, 0.7 and 0.5 for generating sufficient, limited, and short numbers of lots, respectively, and [.] is the closest integer to $\cdot$

Using the data sets, we compared the priority rules to each other and to CPLEX 12.1, which is one of the best commercial optimization software packages. All of the tests were performed on a personal computer with an Intel Core i5 processor operating at a $3.20 \mathrm{GHz}$ clock speed with $4 \mathrm{~GB}$ of RAM. First, the problem instances were solved using CPLEX with a $1 \mathrm{~h}$ time limit. Table 2 lists the test results with CPLEX. CPLEX found optimal and feasible solutions for 98 and 356 problem instances out of the 540, respectively. As the number of orders increased, the problem size increased, and CPLEX hardly found optimal solutions as a result. In addition, as the due dates became tighter and the number of lots decreased, the problems became more difficult to solve, and it became more difficult to find optimal solutions using CPLEX. When the due date was tight or the lots were short, CPLEX could not find optimal solutions for all the instances. For the 200order problem instances, CPLEX failed to find even feasible solutions for $84 \%$, which means CPLEX is not a practical tool for solving real-world lot-order assignment problems.

Table 3 lists the priority-rule test results for 50-order problem instances. In the table, rule performance is represented by two measures: the number of best solutions found by each rule (NB) out of the 20 replication problem instances and the average relative deviation index (ARDI). A relative deviation index (RDI) value for rule A represents how relatively good a solution obtained by rule $\mathrm{A}$ is compared to those obtained by other rules, and it is calculated as $\left(T_{\text {worst }}-T_{\mathrm{A}}\right) /\left(T_{\text {worst }}-T_{\text {best }}\right)$, where $T_{\mathrm{A}}, T_{\text {best }}$, and $T_{\text {worst }}$ are the total tardiness by rule $\mathrm{A}$, along with the best (smallest) and worst (largest) total tardiness values among all the rules, respectively. The RDI value is between 0 and 1 , where 1 means the rule found the best solution among all the rules, and 0 means the opposite. In the table, MDD performed the best in terms of both NB and ARDI. Further, COVERT and ATC found favorable solutions that were considerably close to the best solutions. MOQ showed the worst performance because it does not consider the due date.

Table 4 lists the test results for the 100-order problem instances. These results are similar to those in Table 2. MDD found the greatest number of best solutions, and MDD, COVERT, and ATC showed the best performances in terms of solution quality.

Table 5 lists the test results for the 200-order problem instances. In this test, COVERT and ATC beat MDD in both of the performance measures. Although MDD worked best when the number of lots was sufficiently large to be assigned to orders, its performance deteriorated significantly when there were insufficient lots, and therefore, new lots needed to be released into the production facility. Summarizing the test results in Tables 2-5, MDD, COVERT, and ATC outperformed the other rules. The MDD performance seemed to be affected by the problem characteristics such as the problem size, due-date tightness, and work-in-process level, whereas COVERT and ATC seemed to be robust rules, which guaranteed favorable performances in any case.

To validate the absolute performances of the suggested rules, we compared the suggested rules with CPLEX. For the sake of convenience, we only compared MDD with CPLEX because MDD is one of the best rules. Table 6 lists the results of the comparison between MDD and CPLEX in terms of two measures: average optimality gap (AOG) and average CPLEX gap (ACG). The AOG denotes a percentage of optimal solutions found by MDD for the 20 replication instances in each problem group for which CPLEX also found optimal solutions, and the ACG denotes an average total tardiness gap percentage between the CPLEX and MDD solutions in each problem group. Note that "N.A." in the AOG (ACG) column in the table means that CPLEX failed to find optimal (feasible) solutions for all the problem instances in the problem group, and no optimal (feasible) solutions are available. In the table, the AOG values are close to $100 \%$, 
TABLE 3: Test results of priority rules for 50 -order problems.

\begin{tabular}{|c|c|c|c|c|c|c|c|c|c|c|c|c|c|}
\hline \multirow{2}{*}{$\begin{array}{l}\text { Due date } \\
\text { tightness }\end{array}$} & \multirow{2}{*}{ Number of lots } & \multicolumn{2}{|c|}{ EDD } & \multicolumn{2}{|c|}{ MOQ } & \multicolumn{2}{|c|}{ MDD } & \multicolumn{2}{|c|}{ SLACK } & \multicolumn{2}{|c|}{ COVERT } & \multicolumn{2}{|c|}{ ATC } \\
\hline & & $\mathrm{NB}^{\dagger}$ & $\mathrm{ARDI}^{\ddagger}$ & $\mathrm{NB}^{\dagger}$ & $\mathrm{ARDI}^{\ddagger}$ & $\mathrm{NB}^{\dagger}$ & $\mathrm{ARDI}^{\ddagger}$ & $\mathrm{NB}^{\dagger}$ & $\mathrm{ARDI}^{\ddagger}$ & $\mathrm{NB}^{\dagger}$ & $\mathrm{ARDI}^{\ddagger}$ & $\mathrm{NB}^{\dagger}$ & $\mathrm{ARDI}^{\ddagger}$ \\
\hline \multirow{3}{*}{ Loose } & Sufficient & 17 & 0.99 & 0 & 0.00 & 20 & 1.00 & 15 & 0.99 & 10 & 0.99 & 17 & 0.99 \\
\hline & Limited & 3 & 0.97 & 0 & 0.00 & 20 & 1.00 & 1 & 0.96 & 0 & 0.94 & 1 & 0.98 \\
\hline & Short & 0 & 0.76 & 0 & 0.00 & 13 & 0.99 & 0 & 0.70 & 1 & 0.95 & 6 & 0.99 \\
\hline \multirow{3}{*}{ Normal } & Sufficient & 13 & 0.99 & 0 & 0.00 & 19 & 1.00 & 10 & 0.99 & 6 & 0.99 & 14 & 0.99 \\
\hline & Limited & 0 & 0.80 & 0 & 0.00 & 16 & 0.99 & 0 & 0.73 & 1 & 0.97 & 4 & 0.98 \\
\hline & Short & 0 & 0.23 & 0 & 0.11 & 4 & 0.97 & 0 & 0.11 & 9 & 0.98 & 9 & 0.99 \\
\hline \multirow{3}{*}{ Tight } & Sufficient & 0 & 0.79 & 0 & 0.00 & 15 & 0.99 & 0 & 0.75 & 5 & 0.98 & 1 & 0.98 \\
\hline & Limited & 0 & 0.12 & 0 & 0.26 & 11 & 0.99 & 0 & 0.00 & 6 & 0.99 & 6 & 0.98 \\
\hline & Short & 0 & 0.11 & 0 & 0.68 & 6 & 0.98 & 0 & 0.00 & 11 & 0.99 & 7 & 0.98 \\
\hline \multicolumn{2}{|c|}{ Sum/average } & 33 & 0.64 & 0 & 0.12 & 124 & 0.99 & 26 & 0.58 & 49 & 0.98 & 65 & 0.99 \\
\hline
\end{tabular}

${ }^{\dagger}$ Number of the best solutions found.

*Average relative deviation index.

TABLE 4: Test results of priority rules for 100 order problems.

\begin{tabular}{|c|c|c|c|c|c|c|c|c|c|c|c|c|c|}
\hline \multirow{2}{*}{$\begin{array}{l}\text { Due date } \\
\text { tightness }\end{array}$} & \multirow{2}{*}{ Number of lots } & \multicolumn{2}{|c|}{ EDD } & \multicolumn{2}{|c|}{ MOQ } & \multicolumn{2}{|c|}{ MDD } & \multicolumn{2}{|c|}{ SLACK } & \multicolumn{2}{|c|}{ COVERT } & \multicolumn{2}{|c|}{ ATC } \\
\hline & & $\mathrm{NB}^{\dagger}$ & $\mathrm{ARDI}^{\ddagger}$ & $\mathrm{NB}^{\dagger}$ & $\mathrm{ARDI}^{\ddagger}$ & $\mathrm{NB}^{\dagger}$ & $\mathrm{ARDI}^{\ddagger}$ & $\mathrm{NB}^{\dagger}$ & $\mathrm{ARDI}^{\ddagger}$ & $\mathrm{NB}^{\dagger}$ & $\mathrm{ARDI}^{\ddagger}$ & $\mathrm{NB}^{\dagger}$ & $\mathrm{ARDI}^{\ddagger}$ \\
\hline \multirow{3}{*}{ Loose } & Sufficient & 15 & 0.99 & 0 & 0.00 & 20 & 1.00 & 12 & 0.99 & 7 & 0.99 & 8 & 0.99 \\
\hline & Limited & 4 & 0.98 & 0 & 0.00 & 19 & 1.00 & 1 & 0.96 & 0 & 0.91 & 0 & 0.96 \\
\hline & Short & 0 & 0.53 & 0 & 0.00 & 3 & 0.96 & 0 & 0.45 & 4 & 0.97 & 13 & 0.99 \\
\hline \multirow{3}{*}{ Normal } & Sufficient & 10 & 0.99 & 0 & 0.00 & 19 & 1.00 & 9 & 0.99 & 3 & 0.99 & 7 & 0.99 \\
\hline & Limited & 0 & 0.67 & 0 & 0.00 & 5 & 0.97 & 0 & 0.60 & 9 & 0.99 & 6 & 0.98 \\
\hline & Short & 0 & 0.09 & 0 & 0.38 & 4 & 0.97 & 0 & 0.00 & 12 & 0.98 & 4 & 0.97 \\
\hline \multirow{3}{*}{ Tight } & Sufficient & 0 & 0.80 & 0 & 0.00 & 12 & 0.99 & 0 & 0.77 & 8 & 0.99 & 0 & 0.96 \\
\hline & Limited & 0 & 0.07 & 0 & 0.41 & 7 & 0.98 & 0 & 0.00 & 8 & 0.99 & 5 & 0.98 \\
\hline & Short & 0 & 0.06 & 0 & 0.77 & 8 & 0.98 & 0 & 0.00 & 9 & 0.99 & 3 & 0.98 \\
\hline \multicolumn{2}{|c|}{ Sum/average } & 29 & 0.58 & 0 & 0.17 & 97 & 0.98 & 22 & 0.53 & 60 & 0.98 & 46 & 0.98 \\
\hline
\end{tabular}

${ }^{\dagger}$ Number of the best solutions found.

${ }^{\ddagger}$ Average relative deviation index.

TABLE 5: Test results of priority rules for 200 order problems.

\begin{tabular}{|c|c|c|c|c|c|c|c|c|c|c|c|c|c|}
\hline \multirow{2}{*}{$\begin{array}{l}\text { Due date } \\
\text { tightness }\end{array}$} & \multirow{2}{*}{ Number of lots } & \multicolumn{2}{|c|}{ EDD } & \multicolumn{2}{|c|}{ MOQ } & \multicolumn{2}{|c|}{ MDD } & \multicolumn{2}{|c|}{ SLACK } & \multicolumn{2}{|c|}{ COVERT } & \multicolumn{2}{|c|}{ ATC } \\
\hline & & $\mathrm{NB}^{\dagger}$ & $\mathrm{ARDI}^{*}$ & $\mathrm{NB}^{\dagger}$ & $\mathrm{ARDI}^{\ddagger}$ & $\mathrm{NB}^{\dagger}$ & ARDI $^{*}$ & $\mathrm{NB}^{\dagger}$ & ARDI $^{*}$ & $\mathrm{NB}^{\dagger}$ & $\mathrm{ARDI}^{\ddagger}$ & $\mathrm{NB}^{\dagger}$ & $\mathrm{ARDI}^{\ddagger}$ \\
\hline \multirow{3}{*}{ Loose } & Sufficient & 17 & 0.99 & 0 & 0.00 & 20 & 1.00 & 14 & 0.99 & 5 & 0.99 & 9 & 0.99 \\
\hline & Limited & 1 & 0.92 & 0 & 0.00 & 9 & 0.98 & 0 & 0.89 & 0 & 0.89 & 10 & 0.99 \\
\hline & Short & 0 & 0.11 & 0 & 0.34 & 1 & 0.82 & 0 & 0.01 & 3 & 0.90 & 16 & 0.99 \\
\hline \multirow{3}{*}{ Normal } & Sufficient & 12 & 0.99 & 0 & 0.00 & 20 & 1.00 & 9 & 0.99 & 5 & 0.99 & 2 & 0.99 \\
\hline & Limited & 0 & 0.46 & 0 & 0.00 & 2 & 0.93 & 0 & 0.37 & 7 & 0.97 & 11 & 0.99 \\
\hline & Short & 0 & 0.06 & 0 & 0.81 & 0 & 0.93 & 0 & 0.00 & 8 & 0.99 & 12 & 0.99 \\
\hline \multirow{3}{*}{ Tight } & Sufficient & 0 & 0.81 & 0 & 0.00 & 13 & 0.99 & 0 & 079 & 7 & 0.99 & 0 & 0.94 \\
\hline & Limited & 0 & 0.06 & 0 & 0.60 & 1 & 0.95 & 0 & 0.00 & 19 & 1.00 & 0 & 0.95 \\
\hline & Short & 0 & 0.05 & 0 & 0.93 & 2 & 0.95 & 0 & 0.00 & 15 & 0.99 & 3 & 0.97 \\
\hline \multicolumn{2}{|c|}{ Sum/average } & 30 & 0.50 & 0 & 0.30 & 68 & 0.95 & 23 & 0.45 & 69 & 0.97 & 63 & 0.98 \\
\hline
\end{tabular}

\footnotetext{
${ }^{\dagger}$ Number of the best solutions found.
}

${ }^{\ddagger}$ Average relative deviation index. 
TABLE 6: Comparison between MDD and CPLEX.

\begin{tabular}{|c|c|c|c|c|c|c|c|}
\hline \multirow{2}{*}{$\begin{array}{l}\text { Due date } \\
\text { tightness }\end{array}$} & \multirow{2}{*}{$\begin{array}{l}\text { Number of orders } \\
\text { Number of lots }\end{array}$} & \multicolumn{2}{|c|}{50} & \multicolumn{2}{|c|}{100} & \multicolumn{2}{|c|}{200} \\
\hline & & $\mathrm{AOG}^{\dagger}(\%)$ & $\mathrm{ACG}^{\ddagger}(\%)$ & AOG (\%) & ACG (\%) & AOG (\%) & ACG (\%) \\
\hline \multirow{3}{*}{ Loose } & Sufficient & 100.0 & 0.00 & 100.0 & -11.1 & 100.0 & 0.00 \\
\hline & Limited & 100.0 & -38.5 & 33.3 & -56.1 & N.A. & N.A. \\
\hline & Short & N.A. & -40.7 & N.A. & -29.9 & N.A. & -20.11 \\
\hline \multirow{3}{*}{ Normal } & Sufficient & 93.3 & -11.9 & 91.7 & -35.9 & 100.0 & -7.4 \\
\hline & Limited & N.A. & -44.0 & N.A. & -60.5 & N.A. & N.A. \\
\hline & Short & N.A. & -45.6 & N.A. & -44.4 & N.A. & N.A. \\
\hline \multirow{3}{*}{ Tight } & Sufficient & N.A & -69.3 & N.A. & N.A. & N.A. & N.A. \\
\hline & Limited & N.A & -59.5 & N.A. & -48.2 & N.A. & N.A. \\
\hline & Short & N.A & -62.8 & N.A. & -56.3 & N.A. & N.A. \\
\hline
\end{tabular}

${ }^{\dagger}$ Average optimality gap.

${ }^{\ddagger}$ Average CPLEX gap, where CPLEX gap $=($ total tardiness of MDD - total tardiness of CPLEX $) /($ total tardiness of CPLEX $) \times 100$.

which means the MDD found optimal solutions for most problem instances for which CPLEX found optimal solutions. All ACG values are zero or negative and most of them are less than $-30 \%$, which means the MDD solutions were much better than those obtained by CPLEX. Furthermore, all suggested priority rules required very short CPU times (less than $2 \mathrm{~s}$ even for the 200-order problem instances). Thus, we can argue that the suggested priority rules are considerably more effective than commercial optimization software packages for lot-order assignment.

\section{Conclusions}

In this paper, we showed that we can solve the LOAP by solving the SMTTSP with nonnegative time-dependent processing times and suggested six priority rules for the SMTTSPNNTDPT. The results of computational experiments showed that the priority rules are considerably more effective than CPLEX, with three of the rules (MDD, COVERT, and ATC) being the best. The suggested rules are very simple and can be easily implemented in a manufacturing information system to generate candidate lot-order assignment plans. A production manager can start with the candidate plans to draft a final plan using his or her expertise and experience considering various production conditions. In addition, the suggested rules can be implemented in an order management system, and a sales manager can predict the tardiness of a new order when he or she is attempting to assign a due date to the order after receiving it. In the further research, we will develop more sophisticated scheduling algorithms by using the feature of the nonnegative processing time in the SMTTSP-NNTDPT.

\section{Conflict of Interests}

The authors declare that there is no conflict of interests regarding the publication of this paper.

\section{Acknowledgment}

This work was supported by the Incheon National University (International Cooperative) Research Grant in 2011.

\section{References}

[1] K. Knutson, K. Kempf, J. Fowler, and M. Carlyle, "Lot-to-order matching for a semiconductor assembly and test facility," IIE Transactions, vol. 31, no. 11, pp. 1103-1111, 1999.

[2] J. Fowler, K. Knutson, and M. Carlyle, "Comparison and evaluation of lot-to-order matching policies for a semiconductor assembly and test facility," International Journal of Production Research, vol. 38, no. 8, pp. 1841-1853, 2000.

[3] M. Carlyle, K. Knutson, and J. Fowler, "Bin covering algorithms in the second stage of the lot to order matching problem," Journal of the Operational Research Society, vol. 52, no. 11, pp. 1232-1243, 2001.

[4] T. G. Boushell, J. W. Fowler, A. B. Keha, K. R. Knutson, and D. C. Montgomery, "Evaluation of heuristics for a classconstrained lot-to-order matching problem in semiconductor manufacturing," International Journal of Production Research, vol. 46, no. 12, pp. 3143-3166, 2008.

[5] T. S. Ng, Y. Sun, and J. Fowler, "Semiconductor lot allocation using robust optimization," European Journal of Operational Research, vol. 205, no. 3, pp. 557-570, 2010.

[6] T. W. Wu, "Modular demand and supply pegging mechanism for semiconductor foundry," in Proceedings of the IEEE International Symposium on Semiconductor Manufacturing, pp. 325328, San Jose, Calif, USA, 2003.

[7] J.-Y. Bang, K.-Y. An, Y.-D. Kim, and S.-K. Lim, "A due-datebased algorithm for lot-order assignment in a semiconductor wafer fabrication facility," IEEE Transactions on Semiconductor Manufacturing, vol. 21, no. 2, pp. 209-216, 2008.

[8] J.-G. Kim and S.-K. Lim, "Order-lot pegging for minimizing total tardiness in semiconductor wafer fabrication process," Journal of the Operational Research Society, vol. 63, no. 9, pp. 1258-1270, 2012.

[9] S.-K. Lim, J.-G. Kim, and H.-J. Kim, "Simultaneous orderlot pegging and wafer release planning for semiconductor 
wafer fabrication facilities," International Journal of Production Research, vol. 52, no. 12, pp. 3710-3724, 2014.

[10] T. C. Cheng, Q. Ding, and B. M. Lin, "A concise survey of scheduling with time-dependent processing times," European Journal of Operational Research, vol. 152, no. 1, pp. 1-13, 2004.

[11] T. C. Cheng, C. C. Wu, and W. C. Lee, "Some scheduling problems with sum-of-processing-times-based and job-positionbased learning effects," Information Sciences, vol. 178, no. 11, pp. 2476-2487, 2008.

[12] Y. Yin, D. Xu, K. Sun, and H. Li, "Some scheduling problems with general position-dependent and time-dependent learning effects," Information Sciences, vol. 179, no. 14, pp. 2416-2425, 2009.

[13] Y. Yin and D. Xu, "Some single-machine scheduling problems with general effects of learning and deterioration," Computers and Mathematics with Applications, vol. 61, no. 1, pp. 100-108, 2011.

[14] A. Allahverdi, C. T. Ng, T. C. Cheng, and M. Y. Kovalyov, "A survey of scheduling problems with setup times or costs," European Journal of Operational Research, vol. 187, no. 3, pp. 985-1032, 2008.

[15] Y.-F. Hung, C.-C. Wang, and G.-H. Wu, "Scheduling semiconductor multihead testers using metaheuristic techniques embedded with lot-specific and configuration-specific information," Mathematical Problems in Engineering, vol. 2013, Article ID 436701, 14 pages, 2013.

[16] C. Koulamas, "The single-machine total tardiness scheduling problem: review and extensions," European Journal of Operational Research, vol. 202, no. 1, pp. 1-7, 2010.

[17] A. P. J. Vepsalainen and T. E. Morton, "Priority rules for job shops with weighted tardiness costs," Management Science, vol. 33, no. 8, pp. 1035-1047, 1987.

[18] Y.-D. Kim, "A comparison of dispatching rules for job shops with multiple identical jobs and alternative routeings," International Journal of Production Research, vol. 28, no. 5, pp. 953-962, 1990.

[19] Y.-D. Kim, "A backward approach in list scheduling algorithms for multi-machine tardiness problems," Computers \& Operations Research, vol. 22, no. 3, pp. 307-319, 1995.

[20] D. H. Moon and D. P. Christy, "A simulation study for dynamic scheduling in a hybrid assembly/job shop considering the JIT context," Production Planning and Control: The Management Operations, vol. 9, no. 6, pp. 532-541, 1998.

[21] T. C. Chiang and L. C. Fu, "Using dispatching rules for job shop scheduling with due date-based objectives," International Journal of Production Research, vol. 45, no. 14, pp. 3245-3262, 2007.

[22] H. Emmons, "One-machine sequencing to minimize certain functions of job tardiness," Operations Research, vol. 17, no. 4, pp. 701-715, 1969.

[23] K. R. Baker and J. W. Bertrand, "A dynamic priority rule for scheduling against due-dates," Journal of Operations Management, vol. 4, no. 1, pp. 11-22, 1982.

[24] R. S. Russell, E. M. Dar-El, and B. W. Taylor III, "A comparative analysis of the COVERT job sequencing rule various shop performance measures," International Journal of Production Research, vol. 25, no. 10, pp. 1523-1540, 1987. 


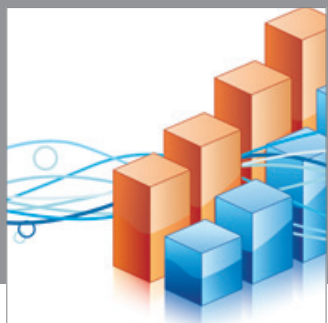

Advances in

Operations Research

mansans

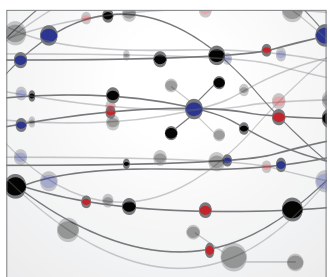

The Scientific World Journal
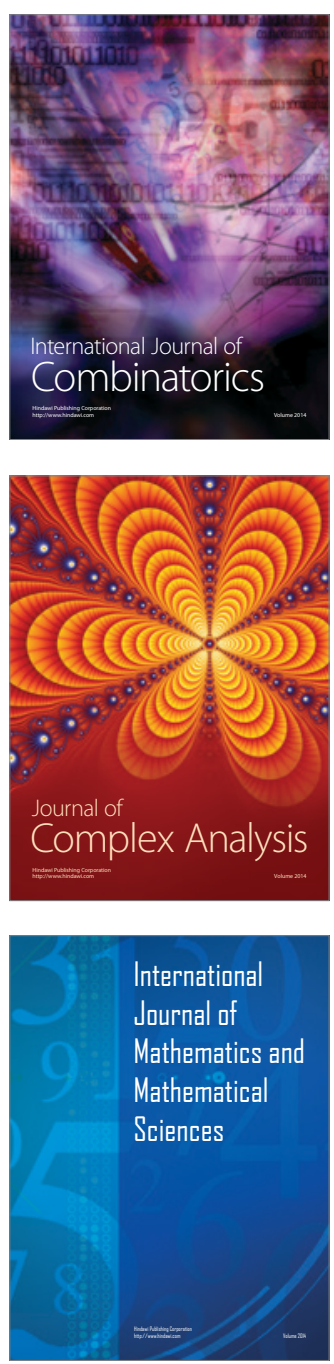
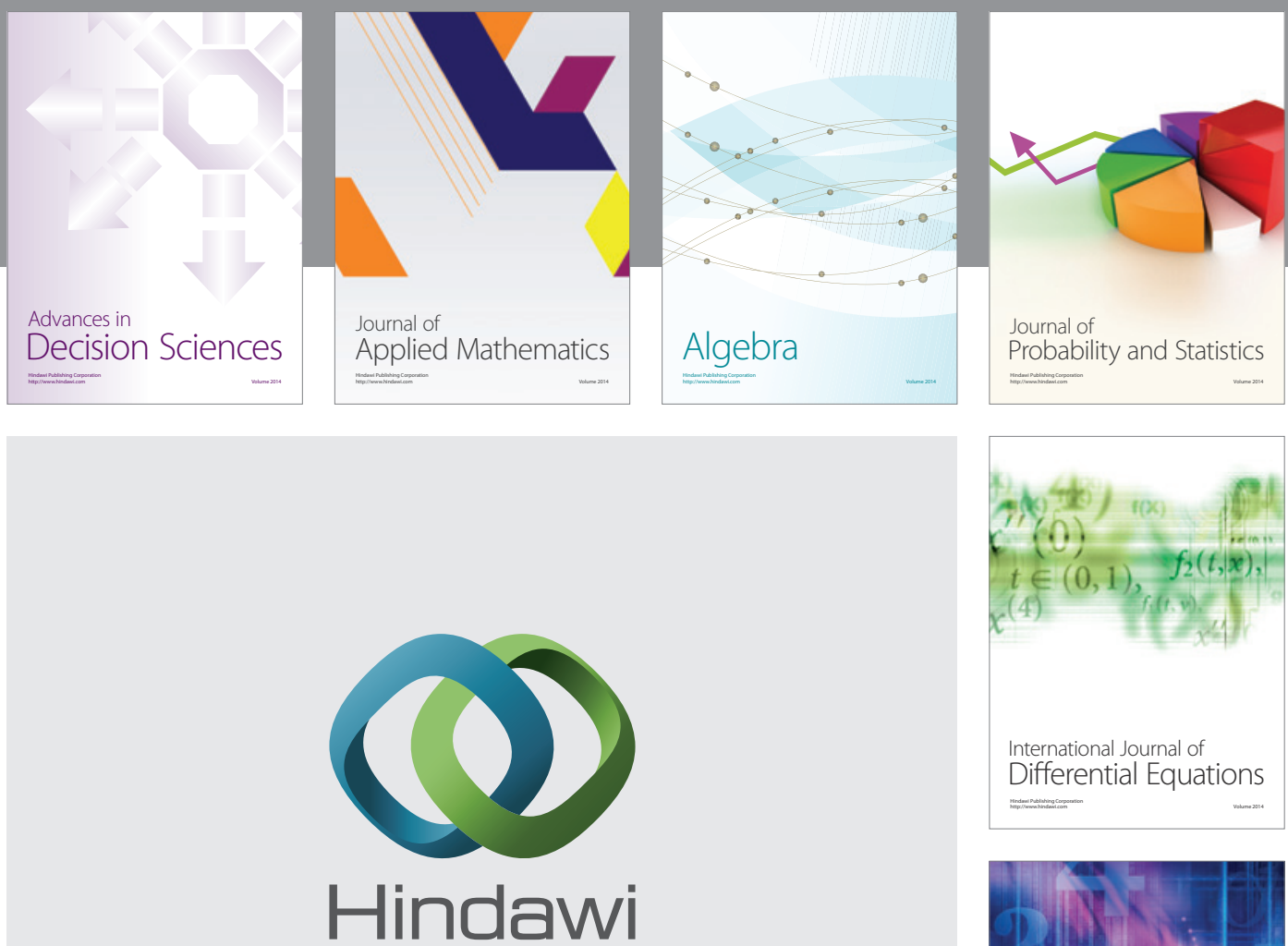

Submit your manuscripts at http://www.hindawi.com
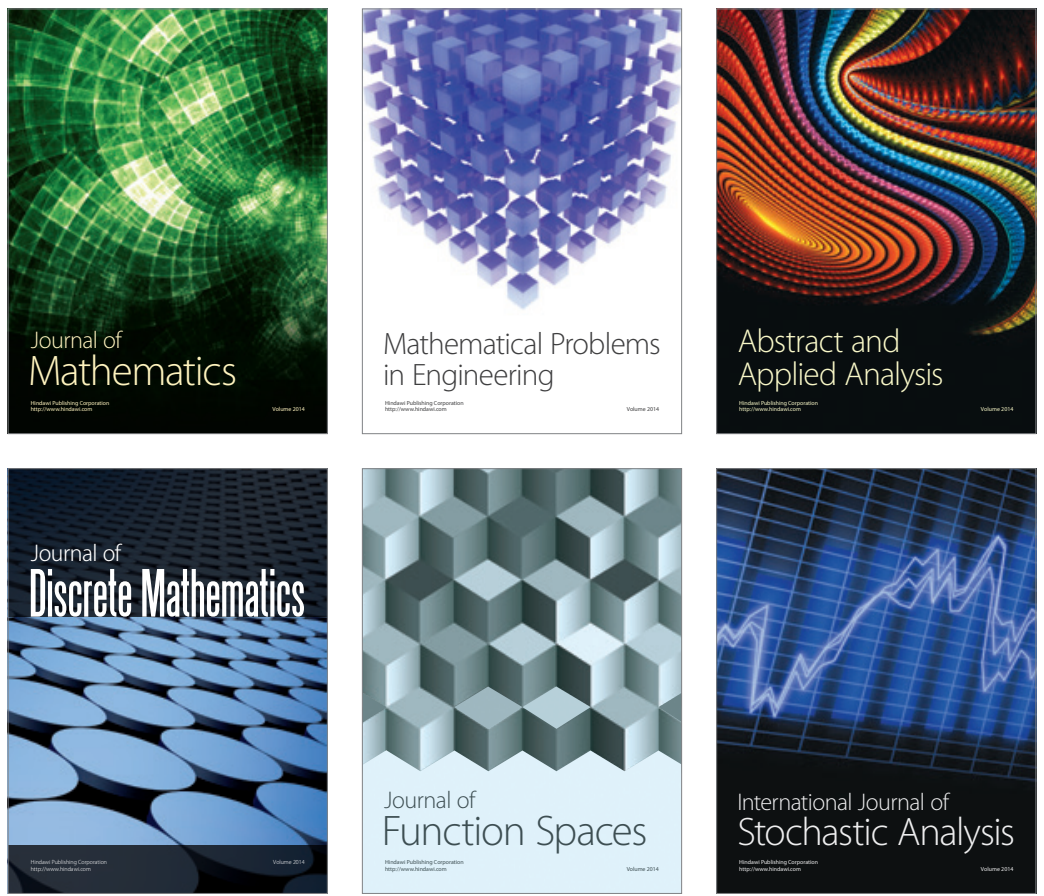

Journal of

Function Spaces

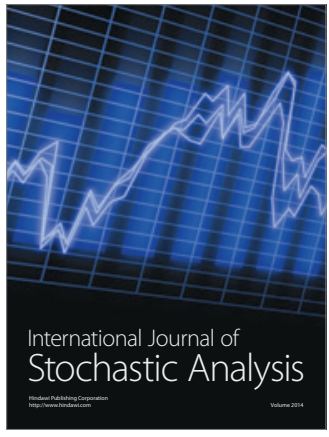

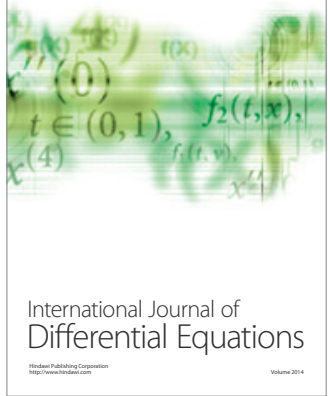
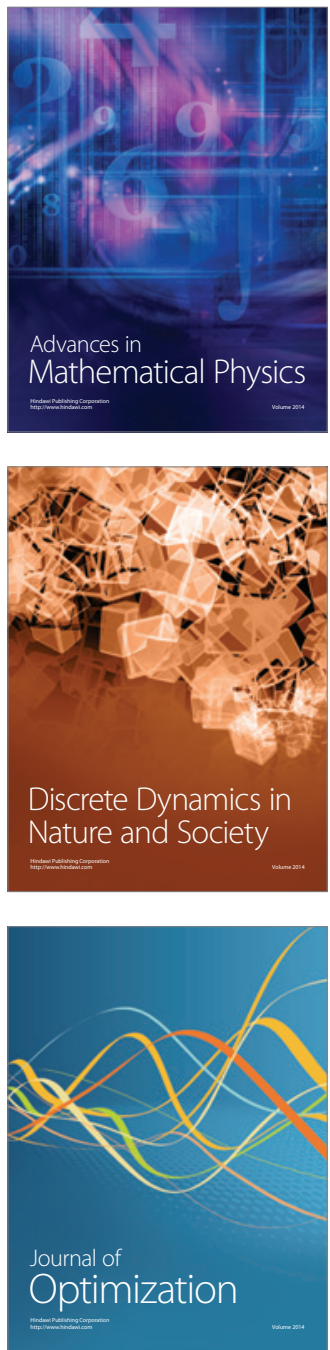